\title{
Comparative Study of Lip Extraction Feature with Eye Feature Extraction Algorithm for Face Recognition
}

\author{
Aakanksha Agrawal \\ Department of CSE \\ RCET, Bhilai \\ CG, India
}

\begin{abstract}
In recent time, along with the advances and new inventions in science and technology, fraud people and identity thieves are also becoming smarter by finding new ways to fool the authorization and authentication process. So, there is a strong need of efficient face recognition process or computer systems capable of recognizing faces of authenticated persons. One way to make face recognition efficient is by extracting features of faces. This paper is to compare the relative efficiency of Lip Extraction and Eye extraction feature for face recognition in biometric devices. Importance of this paper is to bring to the light which Feature Extraction method provides better results under various conditions. For recognition experiments, I used face images of persons from different sets of YALE database. In my dataset, there are total 132 images consisting of 11 persons \& 12 face images of each person.
\end{abstract}

Keywords: face recognition, feature extraction, lip extraction, eye extraction, GLCM, SIFT, SVM

\section{INTRODUCTION}

Biometrics is the study of methods for measuring unique biological and psychological characters of human being that can be used for uniquely recognizing or verifying the individual's identity.

Face Recognition is an identification procedure in which a person is verified based on human traits. Over the past years, Face Recognition gained a lot of attention in the scientific and industrial areas. A number of face recognition algorithms and their extensions, along with distinct measure of success and different accuracy level have been proposed. However, face recognition faces challenging problems in real life applications due to the variation in illumination, position, emotions and expression of the face images. Thus, still better techniques and algorithms are needed to be implemented so as to perform and create better face recognition, better face features extraction, better face detection results, etc.

Feature Extraction is a kind of process for reducing dimensionality so as to represent the interesting image parts with great efficiency as a compact feature vector [16]. This is useful in case of large images.

No one can say which algorithm is best suitable for extracting features. The algorithm selection is dependent on:

1) What exactly is the task it needs to perform?

2) Whether supervised method is needed or unsupervised?

3) Whether Inexpensive method is required or strong computational method is required?

Some numerical programming environments such as MATLAB provide techniques for feature extraction.

Lip Extraction is used as an identifier because the size of the upper and lower lips, furrows, grooves, the distances between the lines and edges vary individualistically. The uniqueness of lip has been proven by the researchers by using colour information and shape analysis. The highlighting advantage in devising the biometric system based on lip recognition is that the data acquisition and handling are simple and hence storing and processing the data becomes an unconstrained task. There are researches carried out in the direction of extracting feature from lip image by shape analysis and color analysis. However lip shape can be changed to a large extent with the change of expression and color of lip is subject to acquisition conditions. Hence, analysis and recognition of an individual by lip-shape and lip-color analysis can only work fine in a constrained scenario. However, the local feature points of a lip image can be exploited as they are invariant to illumination, rotation, scaling and other affine transforms.

\section{LITERATURE REVIEW}

Sambit Bakshi, Rahul Raman, Pankaj K Sa paper proposes that grayscale lip images constitute local features. The claim has been experimentally established by extracting local features applying two techniques viz. SIFT and SURF. The results obtained are enough to establish that unique local features exist in lip images through which an individual can be recognized. [India Conference (INDICON), 2011 Annual IEEE].

Ishvari S. Patel, Apurva A. Desai used Preprocessing techniques like Edge Detection by Canny Method and Height and Width comparison for Lip Contour Detection. This model works effectively and gives around 98\% result for image sequences but we can still improve accuracy of result by extracting perfect lips region. [International Journal of Scientific Research (IJSR), Volume II, Issue V, May 2013].

Zeynep Orman, Abdulkadir Battal, Erdem Kemer recognised that haar-like feature approach is the most used method for face recognition projects (especially for video detection of faces and eyes) in recent years, due to be able to work in real time systems with great performance. [International Journal of Computer Science \& Engineering Survey (IJCSES) Vol.2, No.3, August 2011].

Sasikumar Gurumurty, B. K. Tripathy divided methodology into: Mouth Region Localization and Key point's Extraction 
and Model Fitting. In first and second steps, mouth region and key points are found by using hybrid edges, which combine color and intensity information. In third step, cubic polynomial models are fitted using key points position and hybrid edges. An automatic, robust and accurate lip segmentation method has been presented. This is considered as good result and encourage for its use combined with other biometrics systems.[ I.J. Intelligent Systems and Applications (IJISA), July 2012 in MECS].

B. Sangeetha Devi, V.J.Arul Karthick used two processes for lip recognition. First, face detection by Viola and Jones algorithm. Second, lip detection by morphological operation and five various mouth corner points. Lip biometric can be used to authenticate an individual since the lip is unique. [International Journal of Advanced Research Trends in Engineering and Technology (IJARTET), Vol. II, Special Issue I, March 2015].

Tanmay Rajpathak, Ratnesh Kumar, Eric Schwartz proposed a novel technique for eye detection using color and morphological image processing. Firstly, the skin region is detected using a color based training algorithm and six-sigma technique operated on RGB, HSV and NTSC scales. Further analysis involves morphological processing using boundary region detection and detection of light source reflection by an eye, commonly known as an eye dot. This technique is found to be highly efficient and accurate for detecting eyes in frontal face images. [Florida Conference on Recent Advances in Robotics (FCRAR) 2009].

Rutuja G. Shelke, S.A.Annadate presented a novel approach for Face Recognition and Gender classification strategy using the features of lips. Here feature extraction is carried out by using Principal component analysis (PCA) and Gabor wavelet. Out of two techniques, results of Gabor filter are more accurate and fast because it is having less leakage in time frequency domain. [International Journal of Innovation and Scientific Research (IJISR), Vol 10, No.2, Oct.2014, Innovative Space of Scientific Research Journals (ISSR)].

Noopur Desai, Vidhi Dharia, Ishani Shah, Ghanshyam Prajapati attempts to detect eyes in human images and have implemented eye detection using EyeMap. The proposed approach is divided into two phases. The first phase deals with the detection of face in the image. Once the face is detected, the second phase deals with the detection of eyes in the face detected. The results of the implemented algorithm are quite accurate. [International Journal of Innovative Research in Computer and Communication Engineering (IJIRCCE), Vol. 1, Issue 3, May 2013].

Mayank Vatsa, Richa Singh, Afzel Noore proposes algorithms for iris segmentation, quality enhancement, match score fusion, and indexing to improve both the accuracy and the speed of iris recognition. A curve evolution approach is proposed to effectively segment a nonideal iris image using the modified Mumford-Shah functional. Different enhancement algorithms are concurrently applied on the segmented iris image to produce multiple enhanced versions of the iris image. [IEEE Transactions on Systems, Man and Cybernetics-Part B: Cybernetics].
Himanshu Srivastava described the novel techniques developed to create an Iris Recognition System available. This paper proposed a personal identification using iris recognition system with the help of six major steps i.e. image acquisition, localization, Isolation, normalization, feature extraction and matching and also these six steps consists of minor steps to complete each step. The boundaries of the iris, as papillary and limbic boundary, are detected by using Canny Edge Detector \& Circular Hough Transformation. [International Journal of Engineering Research and Applications (IJERA), Vol. 3, Issue 3, May-Jun 2013].

A. Gebejes, R. Huertas analyzed the dependency of the features with the displacement considered for their computation and explored the possibility of features invariant under changes of the distance between the sample and observation position. Two experiments were performed testing of the effect of the scale on the texture features and the definition of the best displacement value. Authors also suggested that the displacement giving the maximum Contrast (MCD) should be the best as it restricts the feature calculations to surely enclose only one texture element. [Conference of Informatics and Management Sciences (IJCTIC), March, 2013].

\section{PROBLEM IDENTIFICATION}

Keeping a focus on my research work, I found that face images fail to detect Lip Segmentation due to some Color Differences and Light Shadow near lip area and also eye feature extraction can match for two different persons sometimes.

\section{METHODOLOGY OVERVIEW}

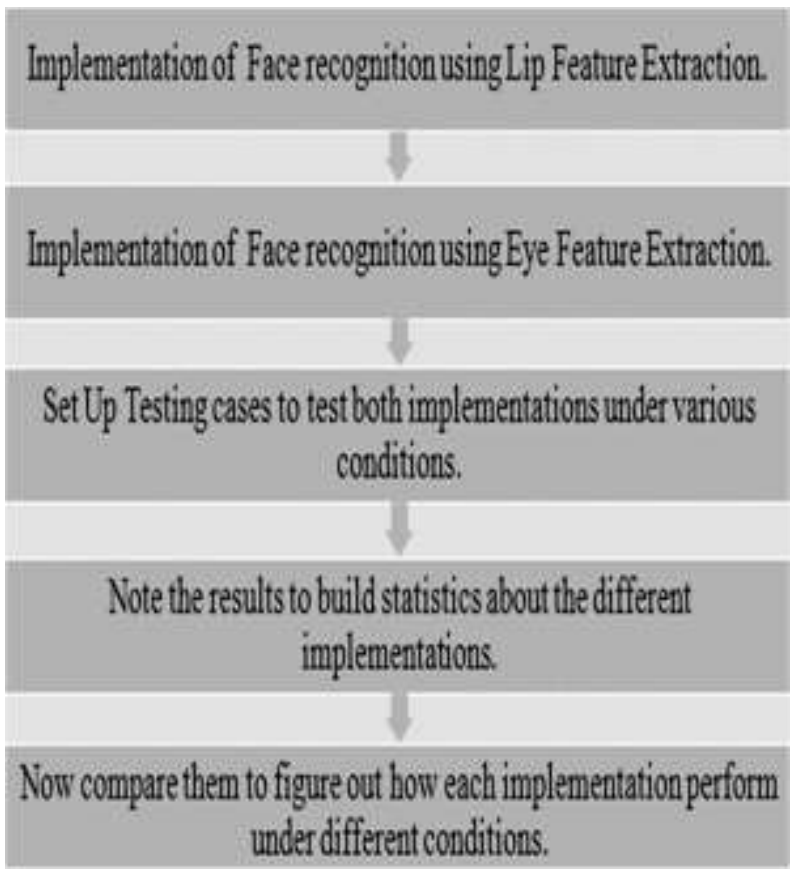

Figure 1. Methodology Overview of this paper

\section{METHODOLOGY}




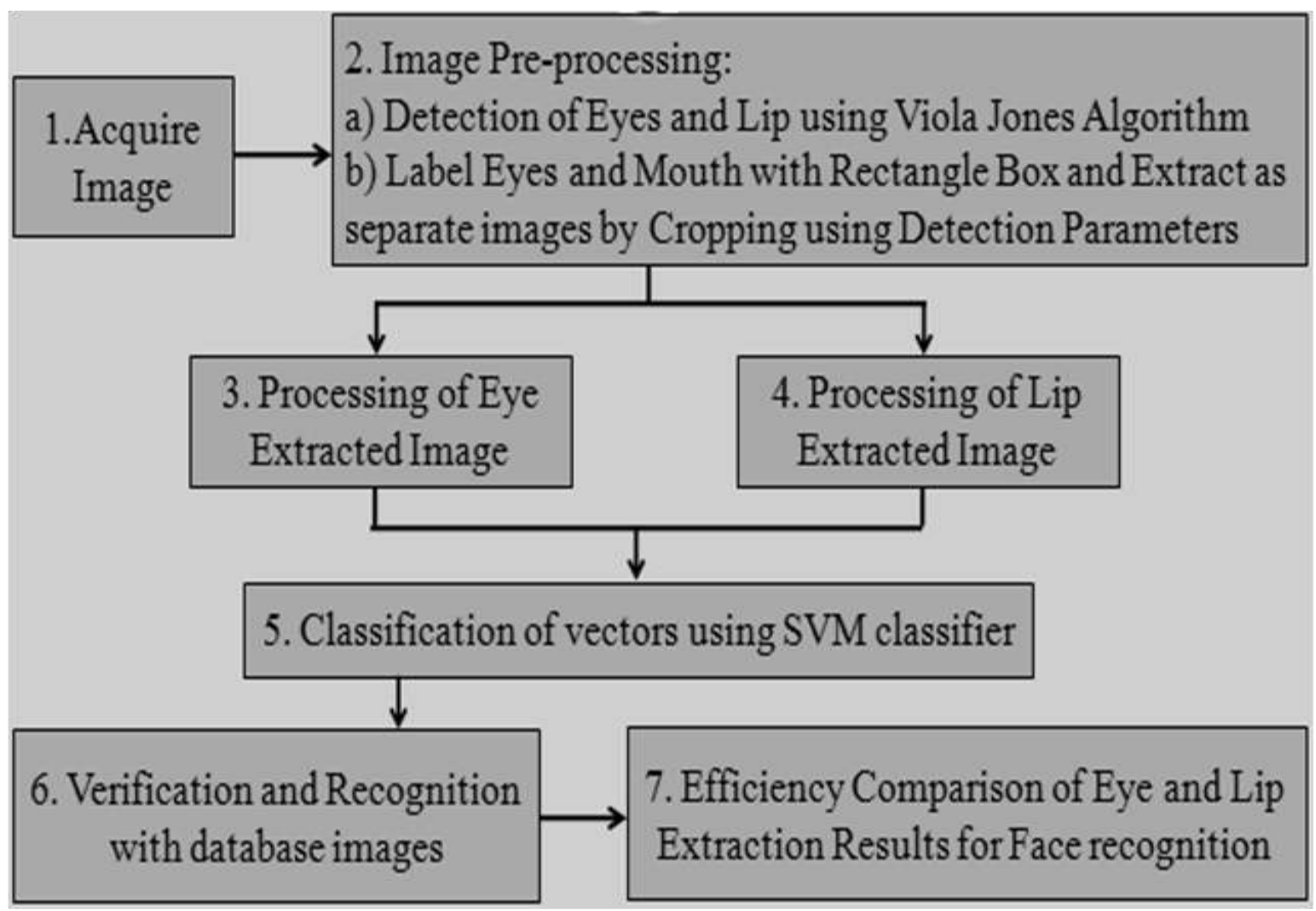

Figure 2. Proposed Methodology of this paper

\subsection{Acquire Image}

5.1.1 Input the image in MATLAB.

5.1.2 Scan the image in MATLAB for further processing.

\subsection{Image Pre-processing}

5.2.1 Detection of Eyes \& Lip using Viola Jones Algorithm

5.2.1.1 Paul Viola and Michael Jone's presented a fast and robust method for face detection which is quicker than any technique with good accuracy. A widely used method for realtime object detection.

5.2.1.2 Training is slow, but detection is very fast.

5.2.1.3 Adaboost is used for object detection. Given a set of weak classifiers:-

None much better than random- Iteratively combine classifiers:

- Form a linear combination to get strong classifier.

- $\quad$ Training error converges to 0 quickly.

- Test error is related to training margin.
5.2.2 Label Eyes \& Mouth with Rectangle box \& Extract as separate images by Cropping using Detection Parameters
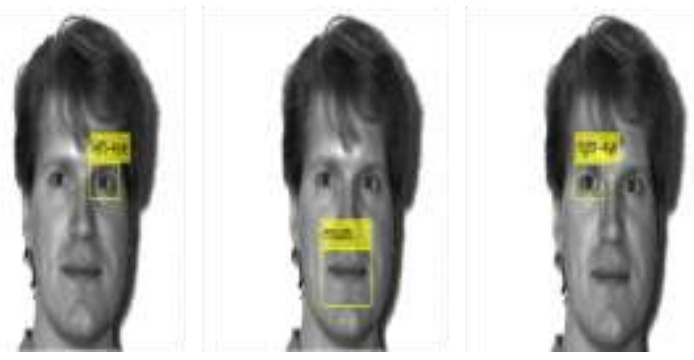

Figure 3. Lefteye, Mouth, Righteye labelled with rectangle box
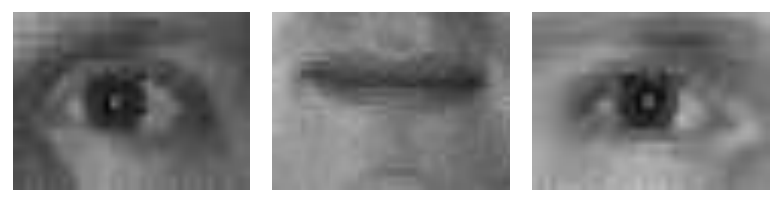

Figure 4. Cropped Lefteye, Mouth, Righteye 


\subsection{Processing of Eye Extracted Image}

\subsubsection{Feature Extraction of Eyes using GLCM:}

Gray Level Co-occurrence Matrix (GLCM) is created from a gray-scale image. Here the texture features of images are extracted and stored in a matrix. GLCM is one of the simplest matrix methods to extract the texture features. GLCM features are extracted for all the images in the database.

A co-occurrence matrix/ distribution, is defined over an image to be the distribution of co-occurring values at a given offset. The co-occurrence matrix is a statistical model and is useful in a variety of image analysis applications such as in biomedical, remote sensing, industrial defect detection systems, etc. Features computed from GLCM are based on the assumption that the texture information in an image is contained in the overall spatial relationship which grey levels of neighbouring pixels have to one another. GLCM contains information about the frequency of occurrence of two neighbouring pixel combination in an image. Although 22 features can be derived from GLCM, usually only some are considered as parameters of importance:

5.3.1.1 Contrast: It is a local grey level variation in the grey level co-occurrence matrix. It can be thought of as a linear dependency of grey levels of neighbouring pixels.

5.3.1.2 Dissimilarity: It is a measure that defines the variation of grey level pairs in an image. It is the closest to Contrast with a difference in the weight. Contrast unlike Dissimilarity grows 'Quadratically'.

5.3.1.3 Entropy: This in any system represents disorder, where in the case of texture analysis, it is a measure of its spatial disorder.

5.3.1.4 Energy: It is a measure of local homogeneity and therefore it represents the opposite of the Entropy. Basically this feature will tell us how uniform the texture is.

5.3.1.5 Prominence: It is a measure of asymmetry. When low, there is a peak in the GLCM matrix around the mean values.

5.3.1.6 Shade: It is a measure of the skewness of the matrix and is believed to follow the concepts of uniformity.

$$
\begin{aligned}
& \text { Contrast }=\sum_{i, j}|i-j|^{2} p(i, j) \\
& \text { Dissimilarity }=\sum_{i, j}|i-j| p(i, j) \\
& \text { Entropy }=-\sum_{i, j} p(i, j) \log (p(i, j)) \\
& \text { Energy }=\sum_{i, j} p(\tilde{z}, j)^{2} \\
& \text { Pro }=\sum_{i=0}^{N_{g}-1} \sum_{j=0}^{N_{g}-1}\left(i+j-u_{x}-u_{y}\right)^{4} p(i, j) \\
& \text { Sha }=\sum_{i=0}^{N_{g}-1} \sum_{j=0}^{N_{g}-1}\left(i+j-u_{x}-u_{y}\right)^{3} p(i, j)
\end{aligned}
$$

Figure 5. GLCM Features Equation

\subsubsection{Feature Extraction of Eyes using ImageJ} Toolbox:

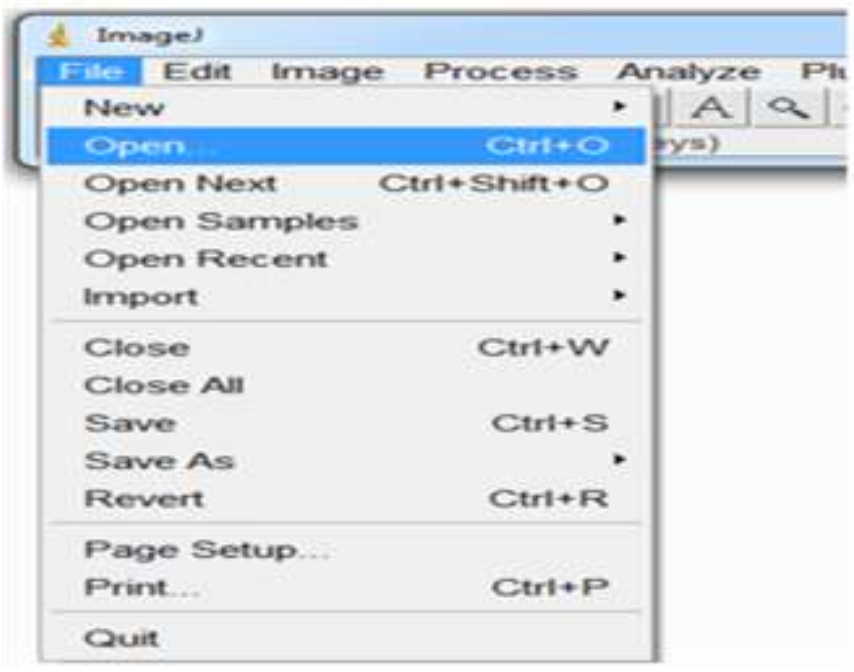

Figure 7. ImageJ Toolbox Screenshot 1

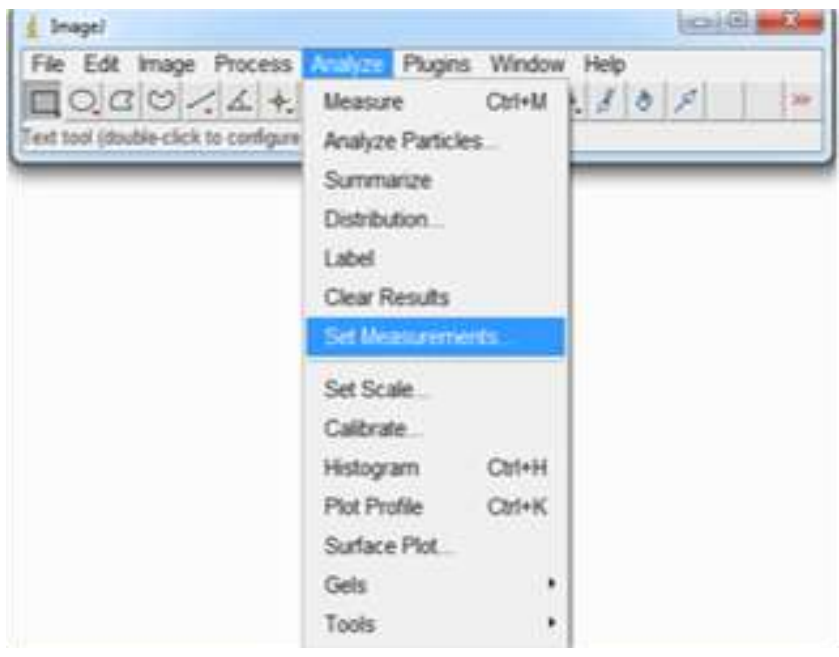

Figure 8.ImageJ Toolbox Screenshot 2

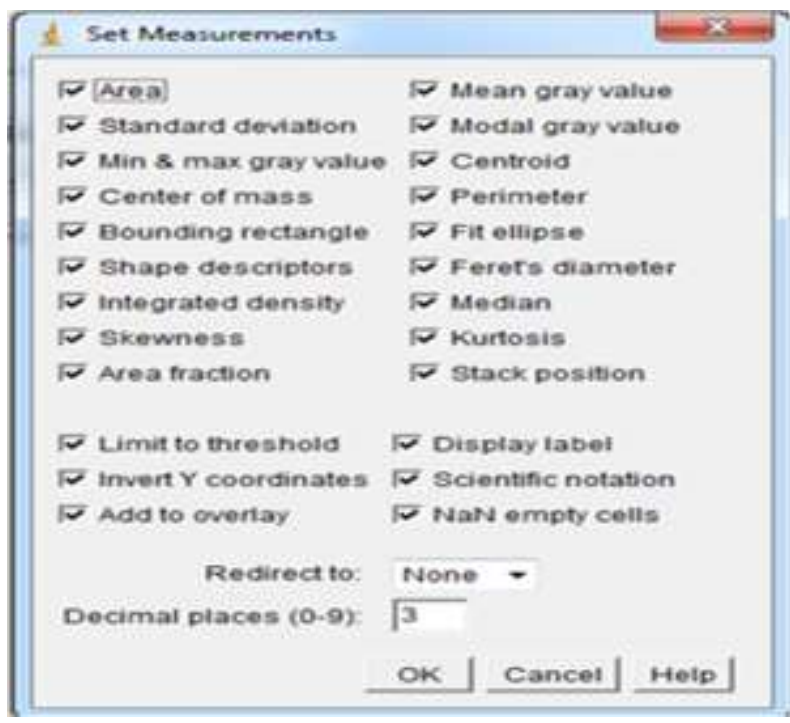

Figure 9. ImageJ Toolbox Screenshot 3 


\subsection{Processing of Lip Extracted Image}

\subsubsection{Feature Extraction of Lips using SIFT:}

Scale Invariant Feature Transform (SIFT) is a method to detect local key points in a scene which are significantly important. SIFT key-points of objects are first extracted from a set of reference images and stored in a database.The technique has three steps:

\subsubsection{Key-point detection by Difference of Gaussian (DoG)} method:

We begin by detecting points of interest, which are termed key-points in the SIFT framework. The image is convolved with Gaussian filters at different scales, and then the difference of successive Gaussian blurred images is taken. Key-points are then taken as maxima/minima of the Difference of Gaussians (DoG) that occur at multiple scales. Once DoG images have been obtained, key-points are identified as local minima/maxima of the DoG images across scales. This is done by comparing each pixel in the DoG images to its neighbours at the same scale and corresponding neighbouring pixels in each of the neighbouring scales. If the pixel value is the maximum or minimum among all compared pixels, it is selected as a candidate key-point.

\subsubsection{128 dimension key-point descriptor computation by} analyzing orientation histogram:

Each key-point is assigned one or more orientations based on local image gradient directions. Previous step found key-point locations at particular scales and assigned orientations to them. This ensured invariance to image location, scale and rotation. Now we want to compute a descriptor vector for each key-point such that the descriptor is highly distinctive and partially invariant to the remaining variations such as illumination, 3D viewpoint, etc. This step is performed on the image closest in scale to the key-point's scale. Although the dimension of the descriptor, i.e. 128 , seems high, descriptors with lower dimension than this don't perform as well across the range of matching tasks and the computational cost remains low due to the approximate method used for finding the nearest neighbour. Longer descriptors continue to do better but not by much and there is an additional danger of increased sensitivity to distortion and occlusion.

5.4.1.3 Key-point matching between two scenes by nearest neighbour method:

SIFT descriptors are invariant to minor affine changes. To test the distinctiveness of the SIFT descriptors, matching accuracy is also measured against varying number of key-points in the testing database, and it is shown that matching accuracy decreases only very slightly for very large database sizes, thus indicating that SIFT features are highly distinctive.

\subsubsection{Applications of SIFT:}

- Robot localization and mapping

- Panorama stitching

- $\quad 3 \mathrm{D}$ scene modelling, recognition and tracking

- 3D SIFT like descriptors for human action recognition

- Analyzing the Human Brain in 3D Magnetic Resonance Images

\subsection{Classification of vectors using SVM classifier}

A Support Vector Machine (SVM) is a classifier formally defined by a separating hyper-plane. In other words, given labeled training data (supervised learning), the algorithm outputs an optimal hyper-plane.

For a linearly separable set of 2D points which belong to one of two classes, find a separating straight line.

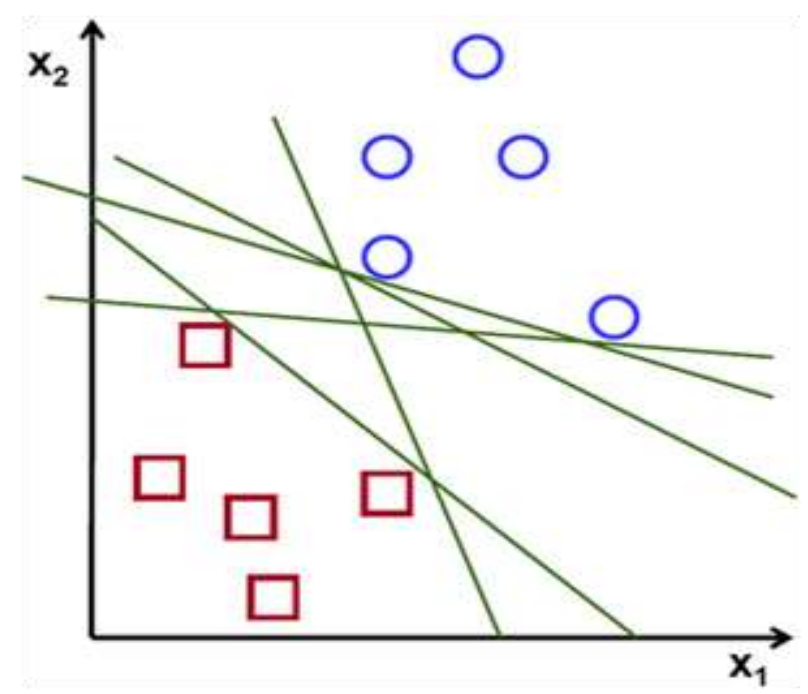

Figure 10. SVM - Separating Straight Line

A line is bad if it passes too close to the points because it will be noise sensitive and it will not generalize correctly. Therefore, our goal should be to find the line passing as far as possible from all points.

Then, the operation of the SVM algorithm is based on finding the hyper-plane that gives the largest minimum distance to the training examples.

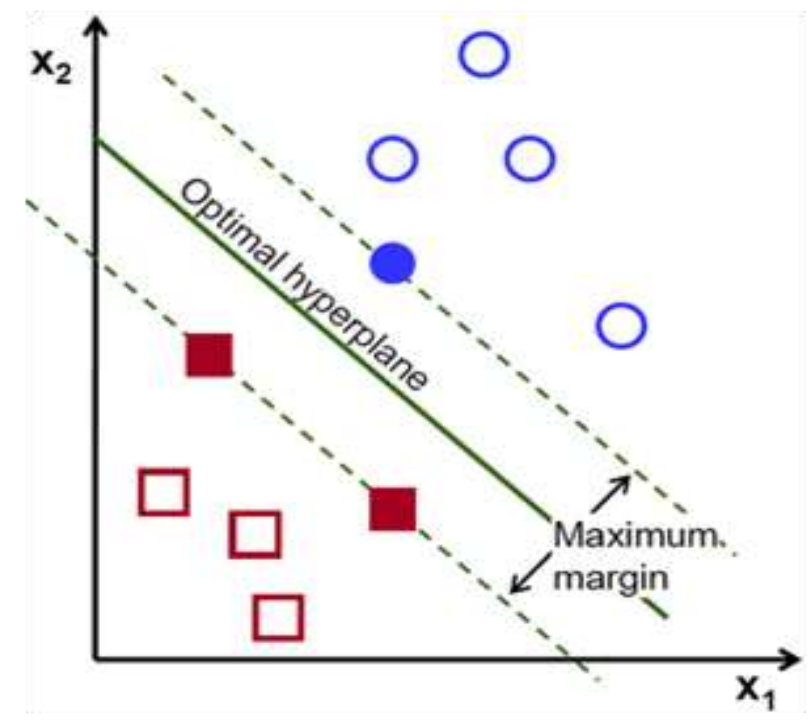

Figure 11. SVM - Optimal Separating Hyper-plane that Maximizes the Margin of the Training Data 


\subsubsection{SVM Algorithm:}

5.5.1.1 A hyper-plane gives a linear discriminant function in dimensions and splits the original space into two halfspaces:

$$
h(x)=w^{T} x+b=w_{1} x_{1}+w_{2} x_{2}+\ldots+w_{d} x_{d}+b,
$$

Where,

$w$ is a $d$-dimensional weight vector,

$b$ is a scalar bias,

Points on the hyper-plane have $h(x)=0$, i.e. the hyper-plane is defined by all points for which $w^{T} x=-b$

5.5.1.2 Given a separating hyper-plane $\mathrm{h}(\mathrm{x})=0$, it is possible to calculate the distance between each point $\mathrm{x}_{\mathrm{i}}$. and the hyperplane by:

$$
\theta_{i}=\frac{y h^{2}\left(x_{i}\right)}{\|x\|}
$$

5.5.1.3 The margin of the linear classifier is defined as the minimum distance of all points to the separating hyper-plane:

$$
\delta^{*}=\min _{x_{i}}\left\{\frac{y_{i} h\left(x_{i}\right)}{\|w\|}\right\}
$$

All points (vectors $\mathrm{x}^{*} \mathrm{i}$ ) that achieve this minimum distance are called the support vectors for the linear classifier. In other words, a support vector is a point that lies precisely on the margin of the classifying hyper-plane.

5.5.1.4 In a canonical representation of the hyper-plane, for each support vector $\mathrm{x}^{*}{ }_{\mathrm{i}}$ with label $\mathrm{y}^{*}{ }_{\mathrm{i}}$ we have:

$$
y_{i}^{*} h\left(x_{i}^{*}\right)=1
$$

Similarly, for any point that is not a support vector, we have:

$$
y h_{2}(x)>1
$$

By definition, it must be farther from the hyper-plane than a support vector. Therefore we have:

$$
y_{i} h\left(x_{i}\right) \geq 1, \forall x_{i} \in D .
$$

The fundamental idea behind SVMs is to choose the hyperplane with the maximum margin, i.e. the optimal canonical hyper-plane.

To do this, one need to find the weight vector $w$ and the bias $b$ that yields the maximum margin among all possible separating hyper-planes, that is, the hyper-plane that maximizes $\mathbf{1} /\|\boldsymbol{w}\|$.

The problem then becomes that of solving a convex minimization problem (notice that instead of maximizing the margin, one can obtain an equivalent formulation of minimizing $\|w\|)$ with linear constraints, as follows:

$$
\begin{array}{cc}
\text { Objective Function } & \text { Linear Constraints } \\
\min \frac{\|w\|^{2}}{2} & y_{i} h\left(x_{i}\right) \geq 1, \forall x_{i} \in D
\end{array}
$$

This minimization problem can be solved using the Lagrange multiplier method, which introduces a Lagrange multiplier $\alpha$ for each constraint:

$$
\alpha_{i}\left(y_{i} h(x)-1\right)=0 \text { with } \alpha_{i} \geq 0
$$

\subsection{Efficiency Comparison of Eye and Lip Extraction Results for Face Recognition}

\section{Performance Evaluation Parameters - Comparison} of:

5.6.1 Accuracy of Lip Extraction \& Eye Extraction

5.6.2 Correct Matches in case of Lip Extraction \& Eye Extraction

5.6.3 Wrong Matches in case of Lip Extraction \& Eye Extraction

\section{RESULTS}

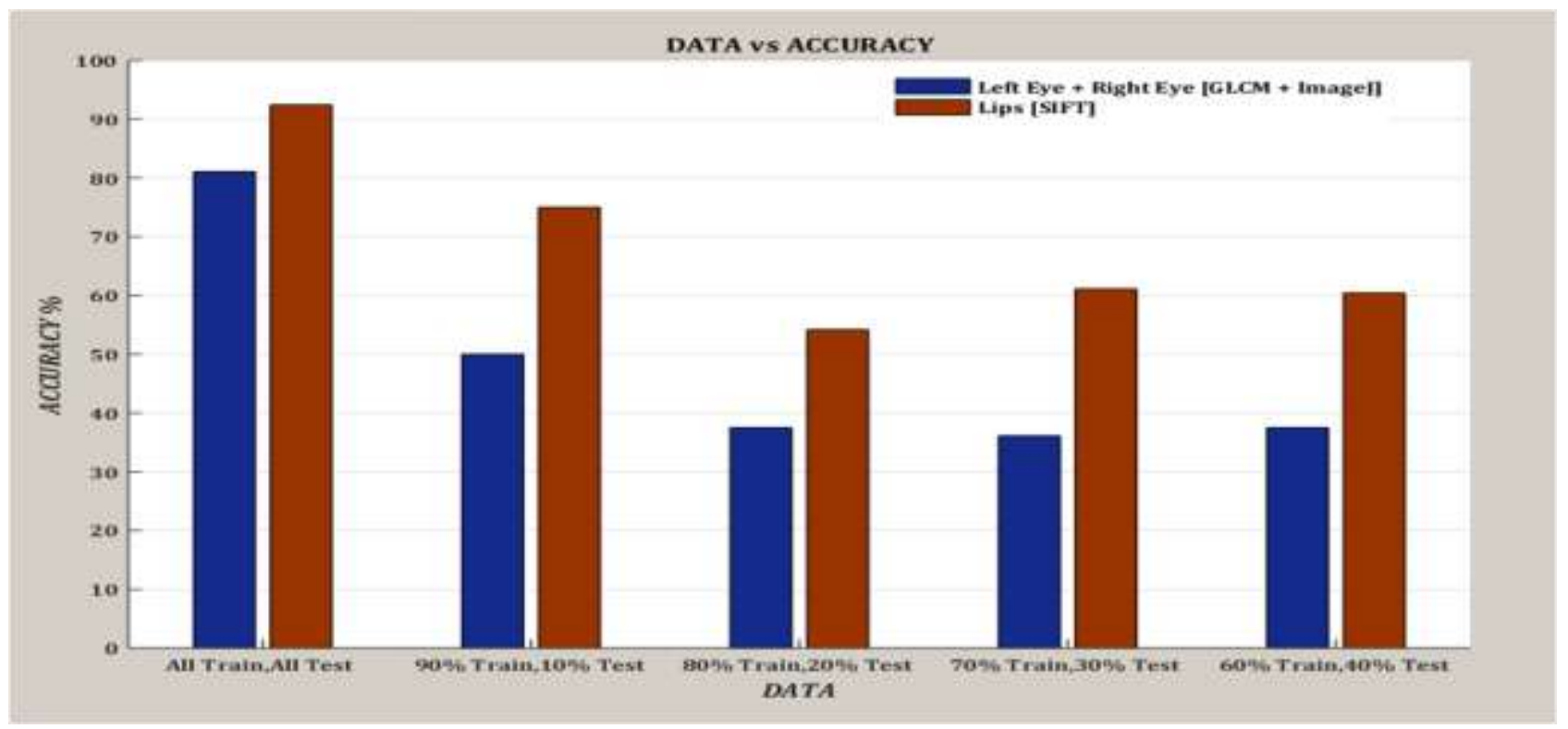

Figure 12. Data vs Accuracy 


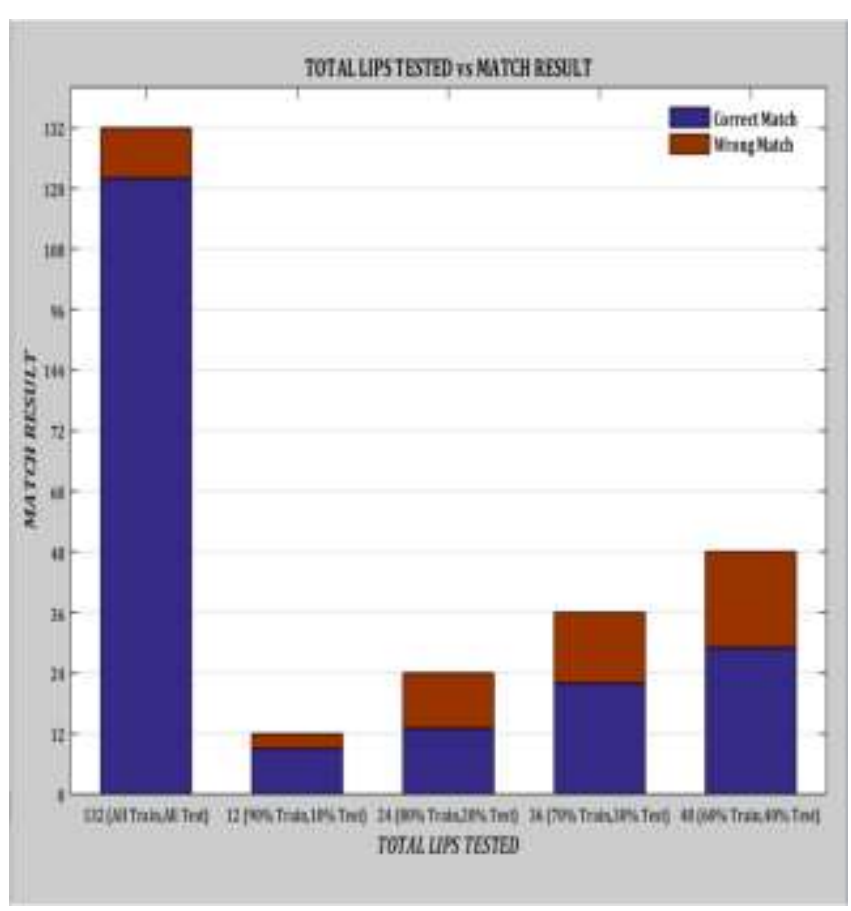

Figure 13. Mouth Performance

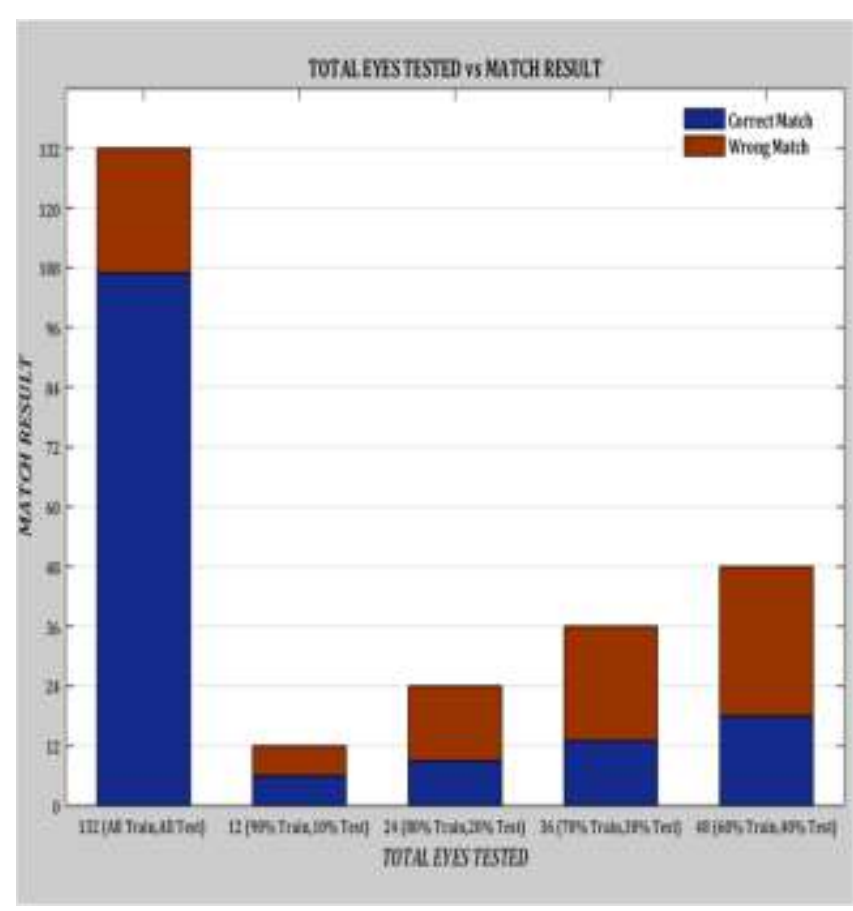

Figure 14. Eyes Performance

Table 1. Data vs Accuracy Plot Results

\begin{tabular}{|c|c|c|c|c|c|c|c|c|}
\hline & \multicolumn{3}{|c|}{$\begin{array}{c}\text { LEFT EYE + RIGHT EYE } \\
\text { METHOD [GLCM + IMAGEJ] }\end{array}$} & \multicolumn{2}{c|}{ MOUTH } & \multicolumn{2}{c|}{ TOTAL DATASET } \\
\hline DATA & Accuracy & Correct & Wrong & Accuracy & Correct & Wrong & TRAIN & TEST \\
\hline $\begin{array}{c}\text { All Train } \\
\text { All Test }\end{array}$ & $81.06 \%$ & 107 & 25 & $92.42 \%$ & 122 & 10 & 132 & 132 \\
\hline $\begin{array}{c}\text { 90\% Train } \\
\text { 10\% Test }\end{array}$ & $50 \%$ & 6 & 6 & $75 \%$ & 9 & 3 & 120 & 12 \\
\hline $\begin{array}{c}\text { 80\% Train } \\
\mathbf{2 0 \%} \text { Test }\end{array}$ & $37.50 \%$ & 9 & 15 & $54.17 \%$ & 13 & 11 & 108 & 24 \\
\hline $\begin{array}{c}\text { 70\% Train } \\
\mathbf{3 0 \%} \text { Test }\end{array}$ & $36.11 \%$ & 13 & 23 & $61.11 \%$ & 22 & 14 & 96 & 36 \\
\hline $\begin{array}{c}\mathbf{6 0 \%} \text { Train } \\
\mathbf{4 0 \%} \text { Test }\end{array}$ & $37.50 \%$ & 18 & 30 & $60.42 \%$ & 29 & 19 & 84 & 48 \\
\hline
\end{tabular}

\section{SCOPE OF FUTURE WORK}

Face Recognition is a very vast and elaborated field. It has no end. As the advancement in science and technology, new techniques will continue developing day-by-day. Today, Lip and Eye extraction are mostly discussed techniques for face recognition purpose but in future many more advance techniques will arise for performing Face Recognition with much more accuracy and efficiency.

\section{ACKNOWLEDGMENTS}

My sincere thanks to all the respected and experienced faculties for their valuable guidance and motivation that always encouraged me to give my full dedication towards a new improvement in the field of science and image processing.

\section{REFERENCES}

[1] B. Sangeetha Devi and V. J. Arul Karthick, "Lip Recognition With Support Vector Machine (SVM) Classifier", International Journal of Advanced Research Trends in Engineering and Technology (IJARTET), Vol. II, Special Issue I, March 2015.

[2] Dr. S. Vijayarani, S. Priyatharsini, "Facial Feature Extraction Based On FPD and GLCM Algorithms", International Journal of Innovative Research in Computer and Communication Engineering, Vol.3, Issue 3, March 2015.

[3] Pankaj K.Sa, S.S. Barpanda, Bansidhar Manjhi, "Region Based Feature Extraction from Non-Cooperative Iris Images", Innovation Syst Software Engg, 2015. 
[4] Sunil Sangve, Nilakshi Mule, "Lip Recognition for Authentication and Security", IOSR Journal of Computer Engineering (IOSR-JCE) Volume 16, Issue 3, Ver. VII, MayJun. 2014.

[5] Rutuja G. Shelke, S. A. Annadate, "Face Recognition and Gender Classification Using Feature of Lips", International Journal of Innovation and Scientific Research (IJISR), Innovative Space of Scientific Research Journals (ISSR), Vol. 10, No. 2, Oct. 2014.

[6] Changcheng Li, Weidong Zhou, Shasha Yuan, "Iris Recognition Based on a Novel Variation of Local Binary Pattern", Springer-Verlag Berlin Hiedelberg, Vis Comput, 2014.

[7] Riddhi Patel and Shruti B. Yagnik, "A Literature Survey on Face Recognition Techniques", International Journal of Computer Trends and Technology (IJCTT), Volume 5, No.4, Nov 2013.

[8] Himanshu Srivastava, "Personal Identification Using Iris Recognition System", International Journal of Engineering Research and Applications (IJERA), Vol 3, Issue 3, May-Jun 2013.

[9] Ishvari S. Patel and Apurva A. Desai, "Lip Segmentation Based on Edge Detection Technique", International Journal of Scientific Research (IJSR), Volume II, Issue V, May 2013.

[10] Noopur Desai, Vidhi Dharia, Ishani Shah, Ghanshyam Prajapati, "Detecting the Location of Eyes on the Front Facial Images Using Two-Phase Method", International Journal of Innovative Research in Computer and Communication Engineering (IJIRCCE), Vol. 1, Issue 3, May 2013.

[11] A. Gebejes, R. Huertas, "Texture Characterization based on GLCM",Conference of Informatics and Management Sciences (IJCTIC), March, 2013.

[12] Jyoti Bedre and Shubhangi Sapkal, "Comparative Study of Face Recognition Techniques", Emerging Trends in Computer Science and Information Technology (ETCSIT2012), International Journal of Computer Applications (IJCA), 2012.

[13] Sasikumar Gurumurty and B. K. Tripathy, "Design and Implementation of Face Recognition System in Matlab Using the Features of Lips", I.J. Intelligent Systems and Applications (IJISA), July 2012 in MECS.

[14] Sambit Bakshi, Rahul Raman, Pankaj K Sa, "Lip pattern recognition based on local feature extraction", India Conference (INDICON), IEEE Annual, 2011.

[15] Zeynep Orman, Abdulkadir Battal, Erdem Kemer, "A Study on Face, Eye Detection and Gaze Estimation", International Journal of Computer Science \& Engineering Survey (IJCSES) Vol.2, No.3, August 2011.

[16] Tanmay Rajpathak, Ratnesh Kumar, Eric Schwartz, "Eye Detection Using Morphological and Colour Image Processing", Florida Conference on Recent Advances in Robotics (FCRAR) 2009.
[17] Mayank Vatsa, Richa Singh, and Afzel Noore, "Improving Iris Recognition Performance Using Segmentation, Quality Enhancement, Match Score Fusion, and Indexing", IEEE Transactions on Systems, Man and Cybernetics- Part B: Cybernetics, Feb 2008.

[18] John Daugman, "New Methods in Iris Recognition", IEEE Transactions on Systems, Man and Cybernetics-Part B: Cybernetics, Vol. 37, No. 5, October 2007.

[19] Duy Nguyen, David Halupka, Parham Aarabi and Ali Sheikholeslami, "Real-Time Face Detection and Lip Feature Extraction Using Field-Programmable Gate Arrays", IEEE Transactions on Systems, Man and Cybernetics-Part B: Cybernetics, Vol. 36, No.4, August 2006.

[20] John Daugman, "How Iris Recognition works", IEEE Transactions on Circuits and Systems for Video Technology, Volume. 14, No. 1, Jan 2004.

[21] Mayank Vatsa, Richa Singh, Afzel Noore, "Improving Iris Recognition Performance Using Segmentation, Quality Enhancement, Match Score Fusion, and Indexing", IEEE Transactions on Systems, Man and Cybernetics-Part B: Cybernetics.

[22] Rafael C. Gonzalez, Richard E. Woods and Steven L. Eddins, "Digital Image Processing Using MATLAB", Second Edition

[23] The MathWorks, Inc., "Image Processing Toolbox", User's Guide, COPYRIGHT 1993-2015

[24] S. N. Sivanandam, S. Sumathi, S. N. Deepa "Neural Network using MATLAB 6.0".

[25] www.google.com 